\title{
Langevin Simulation of Nonlocal Ginzburg-Landau Model for Superconductors in a Magnetic Field
}

\author{
Ayumi FUJITA \\ RIKEN (The Institute of Physical and Chemical Research) \\ Wako, SAITAMA, 351-01, JAPAN
}

(September 18, 2018)

\begin{abstract}
We numerically investigate the phenomenological nonlocal Ginzburg-Landau Hamiltonian for two-dimensional superconductors in a strong magnetic field by Langevin equation. We obtain a regular vortex lattice which is very near to the square lattice. We calculate the structure factor and Abrikosov factor at various temperatures. We also evaluate the specific heat and obtain a cusp which indicates the melting of the vortex lattice.
\end{abstract}

PACS numbers: 74.20.De, 74.60.Ge

The study of the high $T_{c}$ superconductor (HTSC) in a magnetic field has renewed the interests in the mixed state. The most characteristic behavior of this unconventional superconductor is the large superconducting fluctuation, and it is known that the vortex liquid phase appears below the $H_{c 2}$ line in the $H-T$ phase diagram. The melting transition of the vortex lattice has been studied theoretically ever since the discovery of the HTSC. [1] 2] For the two-dimensional case, there has been a large amount of studies which investigate the vortex lattice melting transition of the Kosterlitz-Thouless type which is accompanied by the dissociation of the dislocation pairs. [3., Ex Experimentally, the vortex lattice melting transition is observed as the first order phase transition by revealing a sharp step of the magnetization in both $\mathrm{Bi}_{2} \mathrm{Sr}_{2} \mathrm{CaCu}_{2} \mathrm{O}_{8+\delta}$ and $\mathrm{YBa}_{2} \mathrm{Cu}_{3} \mathrm{O}_{7-\delta}$. [5. 6 .6. The first order melting transition of the vortex lattice is already predicted theoretically from the renormalization group analysis based on Ginzburg-Landau model for three dimensional system. [7] Although it is not yet certainly clear whether the first order melting transition survive in the strong magnetic field phase where the fluctuation effect is very large and the system behaves more like two-dimensional.

On the other hand, the oxide high $T_{c}$ superconductor has a layered structure and it is considered as a quasitwo-dimensional system. It is pointed out that the two-dimensional square lattice structure of $\mathrm{CuO}_{2}$ plane has an effect not only on the symmetry of the order parameter but also on the vortex lattice configuration. [8] Recently many experimental evidences which support the notion that the order parameter of HTSC has $d_{x^{2}-y^{2}}$ symmetry are reported. The expansion of the GL model into a form which deals with the $d$-wave superconductors are thus necessary in order to investigate the vortex state in high- $T_{c}$ cuprates.

In Ref. [9], we have previously investigated a nonlocal GL model starting with following two motivations. One is that the nonlocal GL model has a remarkable similarity to the $n$-component GL model. We find that a parameter of the characteristic range of the nonlocal interaction plays a role of $n$ for the $n$-component GL model. The other one is that because of the shortness of the coherence length of HTSC, modification of the quartic interaction of the order parameter into a nonlocal form is considered to be reasonable.

We introduce a phenomenological nonlocal Hamiltonian,

$$
H[\psi]=\int d^{d} \mathbf{r}\left[\alpha|\psi|^{2}+\frac{\hbar}{2 m}|(-i \nabla-2 e \mathbf{A}) \psi|^{2}\right]+\frac{\beta}{2} \int d^{d} \mathbf{r}_{1} d^{d} \mathbf{r}_{2}\left|\psi\left(\mathbf{r}_{1}\right)\right|^{2} g\left(\mathbf{r}_{1}, \mathbf{r}_{2}\right)\left|\psi\left(\mathbf{r}_{2}\right)\right|^{2},
$$

where the kernel $g\left(\mathbf{r}_{1}, \mathbf{r}_{2}\right)$ represents a nonlocal interaction between $|\psi|^{2}$. For the symmetric case, we choose

$$
g\left(\mathbf{r}_{1}, \mathbf{r}_{2}\right)=\frac{1}{\pi d^{2}} \exp \left(-\frac{\left|\mathbf{r}_{1}-\mathbf{r}_{2}\right|^{2}}{d^{2}}\right)
$$

where $d$ indicates the range of the nonlocal interaction. In Ref. [9] the high temperature series for the free energy is obtained with this Gaussian form nonlocal interaction.

In this study, we investigate numerically the model Hamiltonian Eq. (1) in the case that the kernel $g\left(\mathbf{r}_{1}, \mathbf{r}_{2}\right)$ has a four-fold symmetry with Langevin equation. This model is studied recently in Ref. [10] and they calculate the structure factor using the approximation which is valid at moderately low temperatures. We take the same notation as Ref. [10], and the Fourier transform of $g(\mathbf{r})$ is written as

$$
\tilde{g}(\mathbf{k})=\exp \left[-C\left(\frac{k}{\mu}\right)^{4}(1-\epsilon \cos 4 \theta)\right]
$$


where $\mathbf{k}=k(\cos \theta, \sin \theta)$ and $\mu^{-1}=\sqrt{1 / 2 e B}$ is the magnetic length. The two parameters $C$ and $\epsilon$ determine the amount of the effect of nonlocal interaction on the system. We consider the two-dimensional system in a magnetic field perpendicular to the $x y$-plane. We also take the unit $\hbar=c=k_{B}=1$ and the gauge $\mathbf{A}=(0, x B)$.

In a magnetic field, the order parameter $\psi(\mathbf{r})$ is expanded with the Landau levels. In order to simplify the numerical calculation, we take the lowest Landau level approximation which is valid in a strong magnetic field. Then $\psi(\mathbf{r})$ is written as

$$
\psi(\mathbf{r})=\sum_{q} a_{q} L_{y}^{-1 / 2}\left(\pi / \mu^{2}\right)^{-1 / 4} \exp \left[i q y-\frac{\mu^{2}}{2}\left(x-q / \mu^{2}\right)^{2}\right],
$$

where $q$ is the momentum in $x y$-plane, $a_{q}$ is a complex number and $L_{y}$ is the system size. The Hamiltonian is rewritten with this $\psi$ as

$$
\begin{aligned}
H= & \sum_{q} \alpha_{H}\left|a_{q}\right|^{2}+\frac{\beta}{2 L_{y}^{2}} \sum_{q} \int d^{2} \mathbf{k} \exp \left[-\frac{1}{4 \mu^{2}}\left\{\left(q_{1}-q_{3}\right)^{2}+\left(q_{2}-q_{4}\right)^{2}\right\}-\frac{k_{x}^{2}}{2 \mu^{2}}\right. \\
& \left.-\frac{i}{2 \mu^{2}} k_{x}\left(q_{1}-q_{2}+q_{3}-q_{4}\right)-\frac{C}{\mu^{4}}\left\{(1-\epsilon)\left(k_{x}^{4}+k_{y}^{4}\right)+2(1+3 \epsilon) k_{x}^{2} k_{y}^{2}\right\}\right] \\
& \times a_{q_{1}}^{*} a_{q_{2}}^{*} a_{q_{3}} a_{q_{4}} \delta_{q_{1}+q_{2}, q_{3}+q_{4}} \delta_{q_{1}-q_{3}, k_{y}}
\end{aligned}
$$

In our numerical simulation study, the momentum is discretized as

$$
q=\frac{2 \pi}{L_{y}} n, \quad n=1, \cdots N
$$

where $N$ is the number of vortices in the system with length $L_{x}$ and $L_{y}$,

$$
N=\frac{\mu^{2} L_{x} L_{y}}{2 \pi} .
$$

The Langevin equation for the complex order parameter $a_{q}=A_{q}+i B_{q}$ is given by

$$
\begin{aligned}
& \frac{d A_{q}}{d t}=-\frac{1}{2} \frac{\partial H}{\partial A_{q}}+\eta \\
& \frac{d B_{q}}{d t}=-\frac{1}{2} \frac{\partial H}{\partial B_{q}}+\eta
\end{aligned}
$$

where $\eta$ is the Gaussian white noise. With these Langevin equations we can calculate a set of $a_{q}$ at each time step, and by averaging over the time steps we obtain the statistical average for the values of $a_{q}$. We denote the discretized time step by $\delta$. We rewrite $A_{q}=A(I), B_{q}=B(I)$ where $I$ indicates the discretized momenta $I=1, \ldots, N$. After a time evolution of step $\delta, A(I), B(I)$ becomes $A^{\prime}(I)$ and $B^{\prime}(I)$ which is given by

$$
\begin{aligned}
& A^{\prime}(I)=A(I)-\frac{\delta}{2}\left(2 \alpha_{H} A(I)+S 1\right)+\sqrt{\delta} \eta \\
& B^{\prime}(I)=B(I)-\frac{\delta}{2}\left(2 \alpha_{H} B(I)+S 2\right)+\sqrt{\delta} \eta
\end{aligned}
$$

where

$$
\begin{aligned}
S 1= & \frac{\beta}{2 L_{y}^{2}} \sum_{J_{1}, J_{2}, K_{1}}\left(A\left(J_{1}\right) A\left(J_{2}\right) A\left(I+J_{1}-J_{2}\right)-A\left(J_{1}\right) B\left(J_{2}\right) B\left(I+J_{1}-J_{2}\right)\right. \\
& \left.+2 B\left(J_{1}\right) A\left(J_{2}\right) B\left(I+J_{1}-J_{2}\right)\right) \cos \left(2 \pi K_{1}\left(J_{2}-J_{1}\right) / N\right) h\left(J_{1}, J_{2}, K_{1}\right) \\
S 2= & \frac{\beta}{2 L_{y}^{2}} \sum_{J_{1}, J_{2}, K_{1}}\left(B\left(J_{1}\right) B\left(J_{2}\right) B\left(I+J_{1}-J_{2}\right)-B\left(J_{1}\right) A\left(J_{2}\right) A\left(I+J_{1}-J_{2}\right)\right. \\
& \left.+2 A\left(J_{1}\right) B\left(J_{2}\right) A\left(I+J_{1}-J_{2}\right)\right) \cos \left(2 \pi K_{1}\left(J_{2}-J_{1}\right) / N\right) h\left(J_{1}, J_{2}, K_{1}\right)
\end{aligned}
$$


and

$$
\begin{aligned}
h\left(J_{1}, J_{2}, K_{1}\right)= & \exp \left[-\frac{\pi}{N}\left\{\left(I-J_{2}\right)^{2}+K_{1}^{2}\right\}-C(1-\epsilon)\left(\frac{2 \pi}{N}\right)^{2}\left(K_{1}^{4}+K_{2}^{4}\right)\right. \\
& \left.-2 C(1+3 \epsilon)\left(\frac{2 \pi}{N}\right)^{2} K_{1}^{2} K_{2}^{2}\right] \\
K_{2}= & I-J_{2}
\end{aligned}
$$

We set typical time step $\delta=0.05$ and discard first 40000 steps for the equilibration. We put $N=64$ vortices in the system, and the initial condition is taken as $A(I)=B(I)=0$ for all $I$. We also take the periodic boundary condition in the momentum space.

$$
\left.\begin{array}{l}
A(I)=A(\bmod (I, N)) \\
B(I)=B(\bmod (I, N))
\end{array}\right\} \quad \text { for }|I|>N
$$

We carry out the numerical simulation for various reduced temperatures $y=\left(T-T_{c}\right) / \Delta T$ which is related to $\alpha_{H}$ as

$$
y=\alpha_{H} \sqrt{2 \pi / \beta \mu^{2}} .
$$

Fig. 1 shows a configuration of vortices at temperatures $y=-10$ and $y=-4$. The parameters are taken as $C=0.01$ and $\epsilon=0.5$ in this calculation. We plot $\left\langle|\psi(x, y)|^{2}>\right.$ whose averaging is done over $1.6 \times 10^{5}$ time steps. The white part indicates the large value of $\left\langle|\psi|^{2}\right\rangle$ and the black spots correspond to the vortices. At $y=-10$ we see a regular rhombic vortex lattice structure instead of the triangular lattice. At a temperature $y=-4$, although the magnitude of $\left\langle|\psi|^{2}\right\rangle$ is much lowered the rhombic configuration is still maintained.

The vortex state is probed by the density-density correlation function which is called structure factor. This function is defined in the momentum space as

$$
\Delta(\mathbf{k})=\sum_{q_{i}} a_{q_{1}}^{*} a_{q_{2}}^{*} a_{q_{3}} a_{q_{4}} \exp \left[i \mu^{-2}\left(q_{3}-q_{1}\right) k_{x}\right] \delta_{q_{4}, q_{1}+k_{y}} \delta_{q_{1}+q_{2}, q_{3}+q_{4}}
$$

which is directly related to a neutron diffraction pattern. In the vortex lattice phase

$$
\Delta(\mathbf{k})=\Delta_{0} \delta_{\mathbf{k}, \mathbf{G}}
$$

where $\mathbf{G}$ is the reciprocal lattice vectors. In the case of triangular lattice, the diffraction pattern shows six-fold sharp peaks in the first Brillouin zone. In Fig. 2 we plot $\tilde{\Delta}(\mathbf{k})=\Delta(\mathbf{k}) / \Delta_{0}$ for three different temperatures. In the present nonlocal GL model, the six fold symmetry of the diffraction pattern disappears and a rectangular peaks which correspond to the nearby reciprocal lattice points for the rhombic lattice are obtained. The reciprocal lattice points including the next nearest ones form a stretched hexagon. This configuration almost does not change over the temperature region $-12 \geq y \geq-4$. The height of the peaks, however, become very low at a temperature $y \sim-4$ and this behavior resembles to that of the diffraction pattern obtained by the local GL model at a temperature just below the melting point. In high temperature region $y>-4$, the peaks are totally disappeared. (Note that this temperature is still lower than the $T_{c}$ for the mean field theory.) The ring like diffraction pattern for the vortex liquid phase is not obtained in the present model.

We also calculate the Abrikosov factor

$$
\beta_{A}=\frac{<|\psi(\mathbf{r})|^{4}>}{<|\psi(\mathbf{r})|^{2}>^{2}}
$$

where $\langle\cdots\rangle$ denotes the spatial average. In the vortex lattice state $\beta_{A}$ is given by

$$
\beta_{A}=\sum \tilde{\Delta}(\mathbf{G}) \exp \left(-\mathbf{G}^{2} / 2 \mu^{2}\right)
$$

where the summation is taken over the reciprocal lattice vectors $\mathbf{G}$. For the triangular lattice $\beta_{A}=1.16$ and for the square lattice $\beta_{A}=1.18$. At temperature $y=-12$ we obtain $\beta_{A}=1.20$ which is larger than the value for the square lattice. For the rhombic lattice $\beta_{A}$ is evaluated as

$$
\beta_{A}=\sum_{n, m} \exp \left[-\frac{\pi}{\cos a}\left\{m^{2} \cos ^{2} a+\left(n-m \sin ^{2} a\right)^{2}\right\}\right]
$$


where $a$ is the half of the angle which is made by two adjacent sides. For the case of $\beta_{A}=1.20$, this angle is evaluated as $a=45.84^{\circ}$ which is very near to the value for the square lattice. Above the temperature $y \gtrsim-6$, Abrikosov factor grows rapidly at about $y=-4$ and it converges to the value $\beta_{A}=2.0$ which corresponds to the uncorrelated vortex liquid phase or the normal phase. From this behavior we see that in the nonlocal GL model the melting transition of the vortex lattice takes place at a temperature near $y=-4$.

Next we calculate the specific heat. The specific heat is obtained by taking a second derivative of Eq. (5) with respect to the temperature $\alpha_{H}$.

$$
C=\left\langle\left(\frac{\partial H}{\partial \alpha_{H}}\right)^{2}\right\rangle-\left\langle\frac{\partial H}{\partial \alpha_{H}}\right\rangle^{2}
$$

where

$$
\left\langle\left(\frac{\partial H}{\partial \alpha_{H}}\right)^{2}\right\rangle=\langle\Delta(0)\rangle, \quad\left\langle\frac{\partial H}{\partial \alpha_{H}}\right\rangle^{2}=\frac{1}{N}\left\langle\sum_{q}\left|a_{q}\right|^{2}\right\rangle
$$

In Fig.3 we plot the scaling function for the specific heat $C / \Delta C$ where $\Delta C$ is the specific heat jump at $T_{c}$ in the mean field theory. We obtain a cusp in the scaling function of the specific heat at $y=-4$ at which temperature the Abrikosov factor shows a discontinuous jump. This singularity of the specific heat curve has been previously obtained by the matrix GL model approach with large- $N$ limit where the free energy is calculated up to $O(1 / N)$. [12] In the matrix GL model for the large $N$ limit, the specific heat curve shows remarkable agreement with the usual GL model both in low temperature and high temperature region. In the current model, however, such agreement is lost except the high temperature region.

In summary, we studied a phenomenological nonlocal GL model numerically using the Langevin equations. We obtained the rhombic vortex lattice in the low temperature phase and the corresponding value for the Abrikosov factor $\beta_{A}=1.20$. The cusp in the specific heat suggests that in the nonlocal GL model the superconducting transition reveals a totally different feature from that in the original GL model which shows no singurality at the critical point from the high temperature expansion series study. [11] In order to determine whether the melting transition is discontinuous, detailed studies both with numerical and analytical methods are necessary.

The numerical calculations were made by using the Vector Parallel Processor, Fujitsu VPP500 at RIKEN.

[1] G. Blatter et al., Rev. Mod. Phys. 66,1125 (1994).

[2] D. R. Nelson, Phys, Rev. Lett. 60, 1973 (1988).

[3] S. Doniach and B.A. Huberman, Phys. Rev. Lett. 42, 1169 (1979)

[4] D. S. Fisher, Phys. Rev. B 22, 1190 (1980).

[5] E. Zeldov et al., Nature 375, 373 (1995).

[6] U. Welp et al., Phys. Rev. Lett. 76, 4809 (1996).

[7] E. Brézin, D.R. Nelson and A. Thiaville, Phys. Rev. B 31, 7124 (1985).

[8] K. Maki and H. Won, Ann. Physik 5, 320 (1996).

[9] S. Hikami and A. Fujita, Prog. Theor. Phys. 83, 445 (1990).

[10] J. Yeo and M. A. Moore, Phys. Rev. Lett. (1997)

[11] S. Hikami, A. Fujita and A.I. Larkin, Phys. Rev. B 44, 10400 (1991).

[12] A. Fujita and S. Hikami, Phys. Rev. B 51, 16259 (1995).

FIG. 1. The spatial distribution of $\left\langle|\psi(x, y)|^{2}>\right.$ for the system of $N=64$ vortices. The black spots correspond to the vortices.

FIG. 2. The diffraction pattern at three diffent temperatrues. We plot only the nearby peaks from the origin $\mathbf{k}=0$.

FIG. 3. The scaling function of the specific heat $C / \Delta C$. The results from the different iteration times are shown for the check of equilibration at various temperatures. The cusp at $y=-4$ is enhanced by taking a longer iteration time. 
Fig.1

Title: Langevin Simulation of Nonlocal Ginzburg-Landau Model for Superconductors in a Magnetic Field Author: Ayumi Fujita

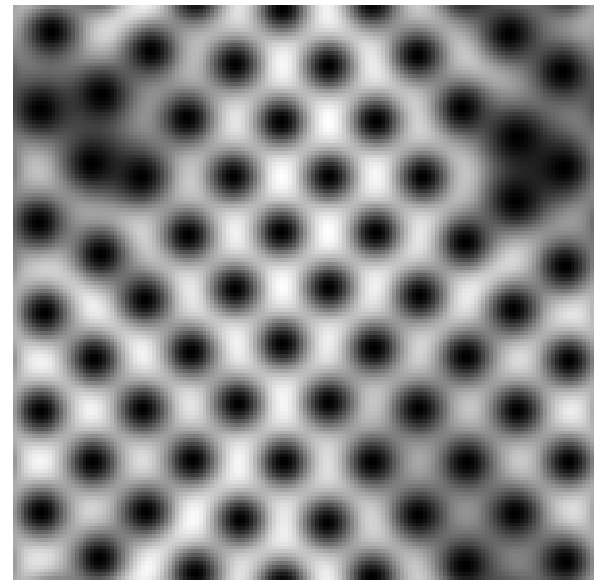

(a) $y=-10$

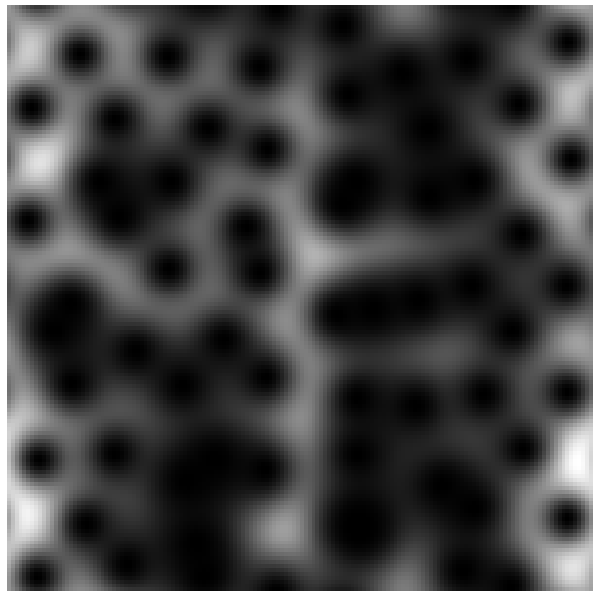

(b) $y=-4$ 
Fig. 2

Title: Langevin Simulation of Nonlocal Ginzburg-Landau model for Superconductors in a Magnetic Field Author:Ayumi Fujita

(a) $y=-10$

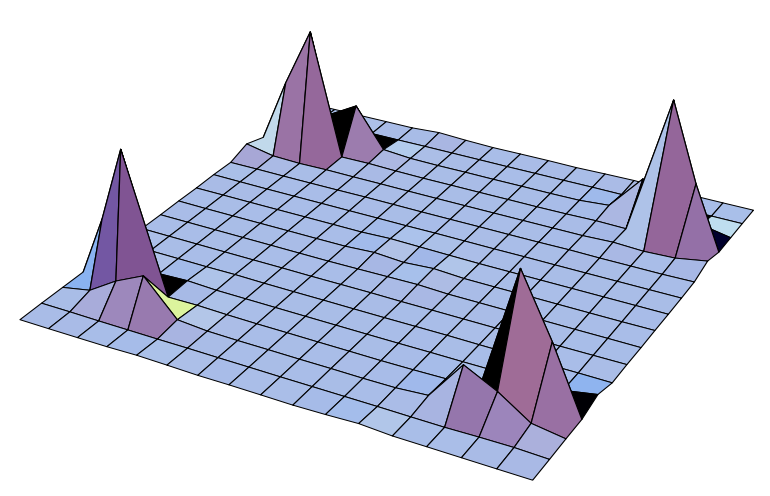

(b) $y=-4$

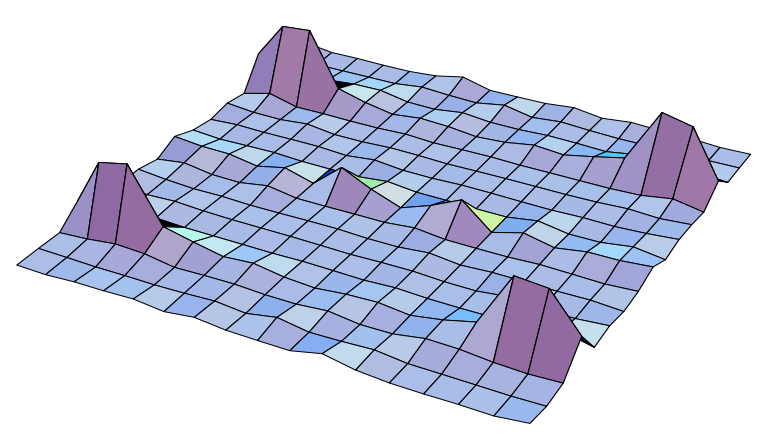

(c) $y=-3.2$

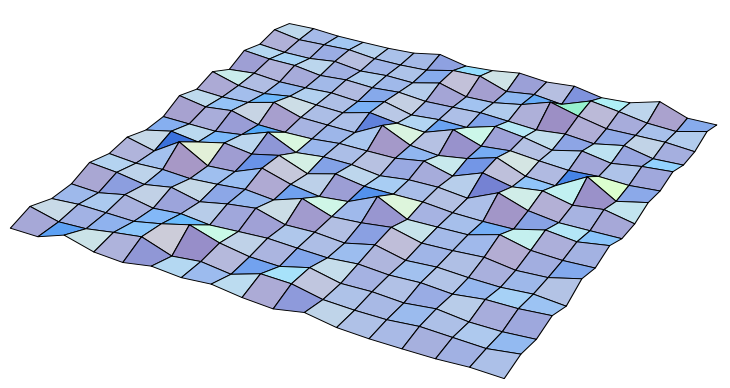


Fig.3

Title: Langevin Simulation of Nonlocal Ginzburg-Landau model for Superconductors in a Magnetic Field Author: Ayumi Fujita

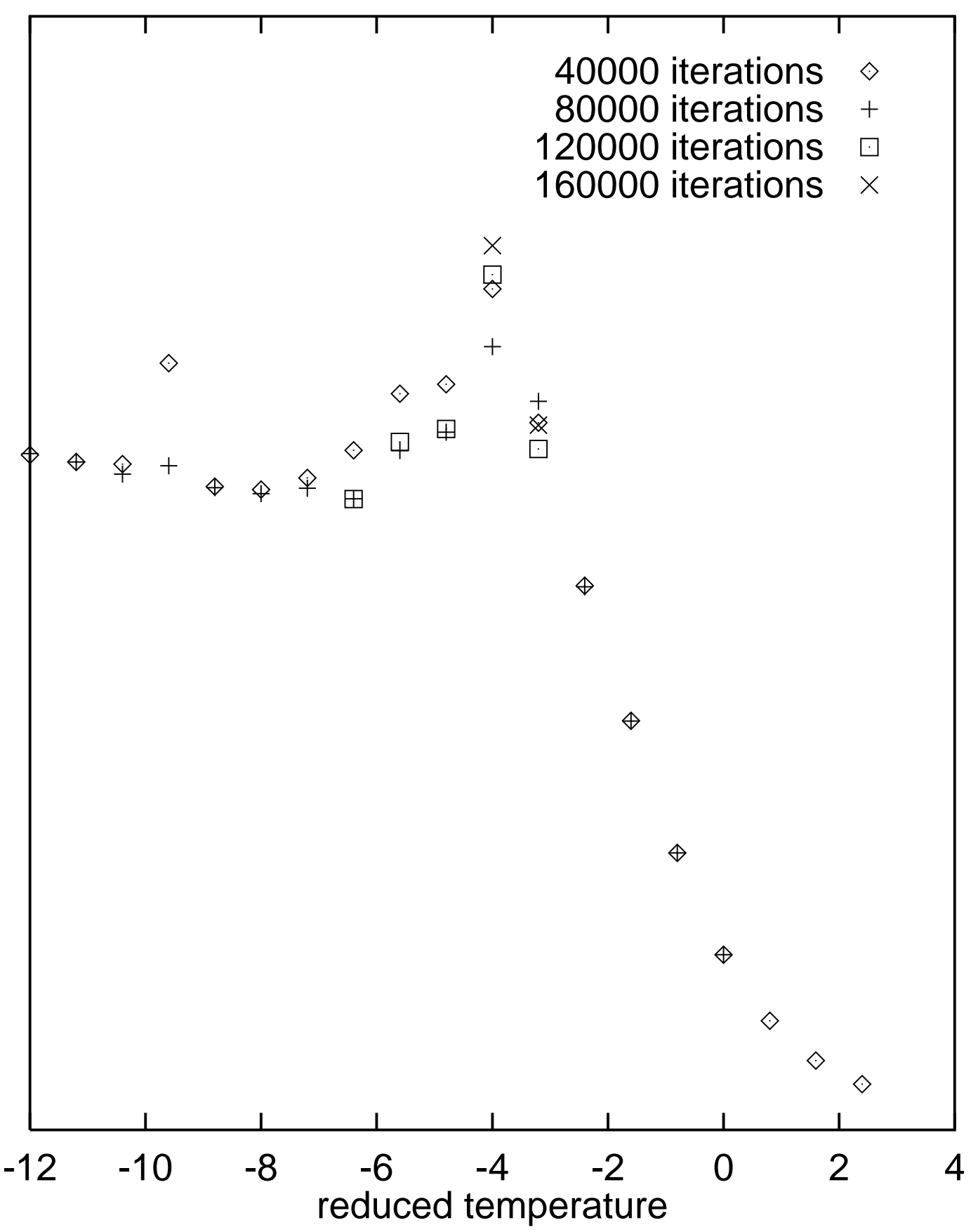

